Peña M.A y Calderón P. (2021): "Evaluación de dos perturbaciones simultáneas en bosques de Arauca ria-Lenga, usando diferencias de indices espectrales”, GeoFocus (Artículos), no 27, p. 5-18. http://dx.doi.org/10.21138/GF.689

\title{
EVALUACIÓN DE DOS PERTURBACIONES SIMULTÁNEAS EN BOSQUES DE ARAUCARIA-LENGA, USANDO DIFERENCIAS DE ÍNDICES ESPECTRALES
}

\author{
aMARCO ANTONIO PEÑA y bPATRICIO CALDERÓN \\ Departamento de Geografía. Universidad Alberto Hurtado \\ Almirante Barroso 10, Santiago, Chile \\ mapena@uahurtado.cly pacaldere@alumnos.uahurtado.cl
}

\section{RESUMEN}

Este estudió evaluó el impacto producido por un incendio forestal y un brote infeccioso encabezado por un pseudo-hongo oomicete, ambos ocurridos en 2015, en el estado de bosques de Araucaria-Lenga de la Reserva China Muerta, ubicada en Los Andes del Sur de Chile. Para esto fue calculado un índice espectral de vigor vegetal sobre un conjunto multitemporal de imágen es Landsat8 , adquiridas bianualmente en fechas de casi aniversario, que luego fue restado entre fechas ex-ante (2013) y ex-post $(2015,2017,2019)$. Los resultados muestran la magnitud relativa de daño y la progresión temporal de ambas perturbaciones, destacando el incendio por su celeridad y agresividad. Si bien las coberturas vegetales afectadas actualmente muestran valores próximos a la recuperación de su biomasa primigenia, se torna necesario ahondar en las posibles variaciones de composición y estructura de estos bosques mediante la incorporación de datos de campo.

Palabras clave: Landsat-8; imágenes ópticas; índices espectrales de vegetación; resiliencia; tipo forestal Araucaria-Lenga.

\section{ASSESSING TWO SIMULTANEOUS DISTURBANCES IN ARAUCARIA-LENGA FORESTS BY SPECTRAL INDEX DIFFERENCES}

\section{ABSTRACT}

This study assessed the impact produced by a wildfire and an infectious outbreak led by an oomycete pseudofungi, both occurred in 2015, on the state of Araucaria-Lenga forests of the China Muerta Reserve, placed in the southern Andes of Chile. To do this, a greenness vegetation spectral index was calculated over a multitemporal set of Landsat-8 images, acquired biannually on nearanniversary dates, which was subject to subtractions between ex-ante (2013) and ex-post dates (2015, 2017, 2019). Results show the magnitude and temporal progression of both disturbances, highlighting the celerity and aggressiveness of the wildfire. Although the affected vegetation land covers currently show values close to the recovery of the primal biomass, the inclusion of field-based data to deepen the possible composition and structure variation s of these forests is needed.

Keywords: Landsat-8; optical images; vegetation spectral indices; resilience; Araucaria-Lenga forest type 
Peña M.A, Calderón P. (2021): "Evaluación de dos perturbaciones simultáneas en bosques de Araucaria-Lenga, usando diferencias de índices espectrales", GeoFocus (Artículos), nº 27, p. 5-18. http://dx.doi.org/10.21138/GF.689

\section{Introducción}

Los bosques dominados por las especies Araucaria araucana (Molina) K. Koch y Nothofagus pumilio (Poepp. et Endl.) Krasser (en adelante bosques del tipo forestal AraucariaLenga), distribuidos en la Cordillera de los Andes del sur de Chile, poseen un importante valor inmaterial y ecológico, producto de su alto endemismo y biodiversidad. Esto, sumado a la multiplicidad de servicios ecosistémicos que brindan (e.g., depuración del aire, balance climático, secuestro de carbono), ha llevado a su preservación bajo la figura de Reservas por parte del Sistema Nacional de Áreas Silvestres Protegidas por el Estado de Chile (CONAF 2014, Figueroa-Burdiles y Vergara-Pinto 2018). Adicionalmente, la Araucaria ha sido declarada Monumento Nacional por el Estado chileno y categorizada en "peligro de extinción" por la Unión Internacional para la Conservación de la Naturaleza (UICN). En tanto la Organización de las Naciones Unidades para la Educación, la Ciencia y la Cultura (UNESCO), ha incorporado a Reservas de este tipo forestal a la Red Mundial de Reservas de la Biosfera (CONAF 2014, Vélez et al. 2018). A pesar de estas acciones de conservación, durante las últimas décadas los bosques de Araucaria-Lenga chilenos han estado expuestos a varias perturbaciones, en especial de orden biótico, que han alterado tanto su estructura como composición vegetal a través del tiempo, interrumpiendo con esto su sucesión ecológica y contribuyendo consecuentemente, a su degradación (González y Veblen 2006, Raffaele et al. 2014, Urrutia et al. 2018).

La zona climática centro-sur de Chile ha experimentado en los últimos 10 años un a mega sequía que ha propiciado el aumento en frecuencia e intensidad de los incendios forestales (De la Barrera y Ruíz 2017, González et al. 2018), incidiendo en la resiliencia de ecosistemas con alto valor ecológico, como lo son los bosques de Araucaria-Lenga (Fuentes-Ramírez et al. 2018). Lo anterior, a pesar de la adaptabilidad que éstos muestran frente a regímenes de fuego superficiales frecuentes e incendios severos poco frecuentes (Urrutia et al. 2018). En efecto, esta mega sequía ha influido en los agotamientos parciales de carbono de las Araucarias, debilitándolas fisiológicamente y predisponiéndolas para un ataque severo y asociativo de estresores bióticos (Vélez et al. 2018). Ante déficit hídrico, esta especie activa la reacción del ácido abscísico (ABA), cerrando sus estomas para conservar agua y generar una homeostasis temprana en la hoja, lo que la protege frente a una eventual cavitación o "falla hidráulica" (Lusk 2001). Si bien este mecanismo confiere a las Araucarias adaptabilidad a condiciones de penumbra y sequía breves, la prolongación de esta situación las expone a distintos grados de patogenicidad asociadas a síntomas como desecación, necrosis acrópeta parcial, defoliación, resinosis, etc., como resultado a largo plazo del desbalance en sus reservas de carbono, conocido como "hambruna de carbono" (Vélez et al. 2018).

La teledetección para la observación terrestre tiene un rol crucial en la evaluación de las dinámicas forestales afectadas por perturbaciones abruptas o graduales, aportando con la obtención y análisis de datos sinópticos y periódicos acerca del estado de las coberturas vegetales (Banskota et al. 2014). Tales datos son adquiridos típicamente por sensores remotos ópticos transportados por satélites y representados en la forma de imágenes planimétricas, cuyos pixeles almacenan los comportamientos espectrales de dichas coberturas (Chuvieco 1995). El análisis de estos comportamientos usando métodos de teledetección y datos auxiliares integrados en una herramienta del tipo SIG (sistema de información geográfica), permite comprender y modelar los patrones espaciales y temporales de una perturbación dada, como podría ser un incendio o una enfermedad forestal, facilitando con ello su manejo y monitoreo ex-post (Hirschmugl et al. 2017). Dentro de la diversidad de métodos plausibles para acometer lo anteriormente expresado, destaca la formulación de índices espectrales de vegetación, que resultan de combinar aritméticamente bandas espectrales de la imagen (i.e., muestreos de longitudes de onda espectral) sensibles a alguna propiedad fisicoquímica del follaje contenido 
Peña M.A y Calderón P. (2021): "Evaluación de dos perturbaciones simultáneas en bosques de Arauca ria-Lenga, usando diferencias de índices espectrales", GeoFocus (Artículos), no 27, p. 5-18. http://dx.doi.org/10.21138/GF.689

en sus píxeles, sirviendo como proxies para caracterizar el estado de una cobertura vegetal (Jones y Vaughan 2010, Xue y Su 2017).

La vegetación sana se caracteriza por absorber mucha radiación solar en las longitudes de onda espectral del rojo, debido a la presencia de clorofila foliar, a la vez de reflejar mucha radiación solar en las longitudes de onda espectral del infrarrojo cercano, debido a la estructura interna de la hoja (Jones y Vaughan 2010). De esta manera, un distanciamiento amplio entre los valores de ambas bandas para un píxel dado de la imagen, es indicativo de una mayor vigorosidad o salud de la cobertura vegetal contenida en este, siendo este principio de contraste espectral la base para la formulación de gran parte de los índices espectrales de verdor o vigor vegetal, como el NDVI (normalized difference vegetation index). Este y otros índices similares, han sido profusamente empleados para evaluar la severidad de daño y el ritmo de recuperación de bosques afectados por diferentes estresores bióticos y abióticos, siendo su resta entre fechas ex-ante y ex-post una de las aproximaciones metodológicas más frecuentemente usadas para detectar y evaluar la magnitud de cambio inducida por la perturbación (Kushla y Ripple 1998, Chuvieco et al. 2002, Frolking et al. 2009, Townsend et al. 2012).

El presente estudio evaluó el estado de bosques del tipo Araucaria-Lenga recientemente perturbados al interior de la Reserva China Muerta, Región de la Araucanía, Chile, mediante la resta entre fechas ex-ante y ex-post de un índice espectral de vigor vegetal derivado desde imágenes Landsdat-8. La Reserva registra la ocurrencia reciente y simultánea de dos grandes perturbaciones fuertemente asociados a la condición de mega sequía imperante en la zona. Por un lado, un incendio forestal, ocurrido entre el 15 de marzo y el 6 de abril de 2015 (FuentesRamírez et al. 2018) y por otro, una infección de las Araucarias ocasionada por varios patógenos, en particular un pseudo-hongo oomicete (Phytophthora spp.), detectada conjuntamente por autoridades de Chile y Argentina a finales del 2015 (Vélez et al. 2018). Se espera que la evaluación del impacto generado por ambas perturbaciones contribuya al monitoreo de estos valiosos bosques y al encausamiento más eficiente de acciones de manejo y restauración de sus ecosistemas.

\section{Materiales, datos y métodos}

\section{1. Área de estudio}

La Reserva Forestal China Muerta se emplaza en la Cordillera de Los Andes perteneciente a las Comunas de Melipeuco y Lonquimay, Región de la Araucanía, Chile. Su ubicación geográfica corresponde a $\operatorname{los} 38^{\circ} 50^{\prime} 00^{\prime}$ ' $\mathrm{S}$ y $71^{\circ} 30^{\prime} 00^{\prime}$ ' $\mathrm{O}$, y su superficie alcanza los $85,33 \mathrm{~km}^{2}$ (Figura 1). Se inserta en un clima templado-cálido de acuerdo a la clasificación de Köppen, reconociéndose dos subregiones; la primera asociada a una formación vegetal de bosques caducifolios Altoandino, y la segunda asociada a una formación de bosques caducifolios mixtos de la Cordillera de los Andes (Gajardo 1994). Estas subregiones generan un paisaje irregular y ampliamente diverso, dominado por varias especies del género Nothofagus, en especial Lenga, además de Ciprés de la cordillera (austrocedrus chilensis (D. Don) Pic. Serm. et Bizarri), Tepa (laureliopsis philippiana (Looser) Schodde) y Araucaria o Pehuén. La Reserva se inscribe en un territorio montañoso accidentado y escarpado, con presencia de algunas colinas y planicies de valle, distribuyéndose a lo largo de un rango altitudinal de entre 700 y 2000 m.s.n.m. (CONAF 2014). 
Peña M.A y Calderón P. (2021): "Evaluación de dos perturbaciones simultáneas en bosques de Arauca ria-Lenga, usando diferencias de indices espectrales", GeoFocus (Artículos), no 27, p. 5-18. http://dx.doi.org/10.21138/GF.689

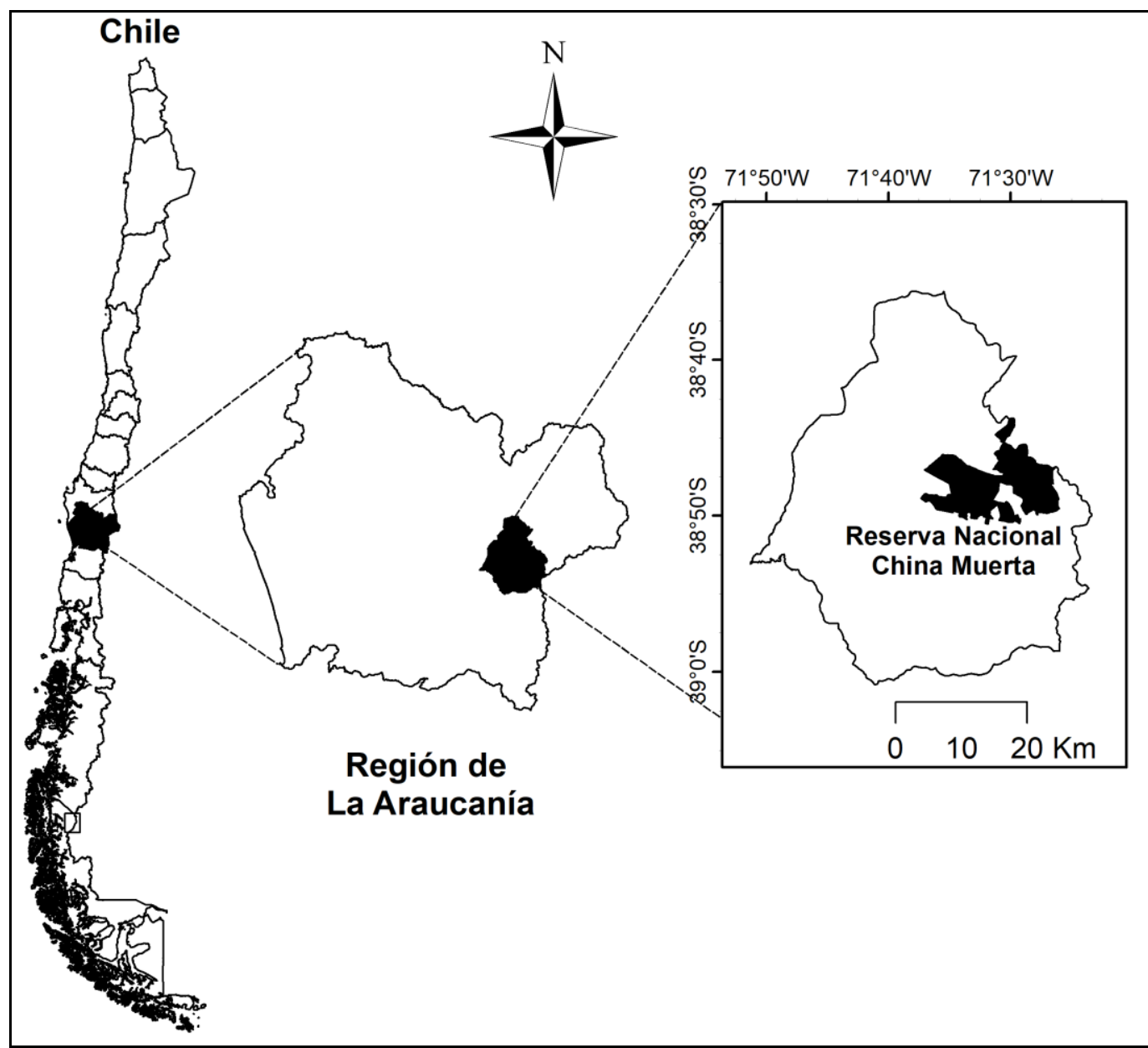

Figura 1. Localización del área de estudio Reserva Nacional China Muerta, ubicada en la comuna de Melipeuco (derecha), Región de la Araucanía (izquierda), Chile.

Fte.: Urrutia et al. (2018).

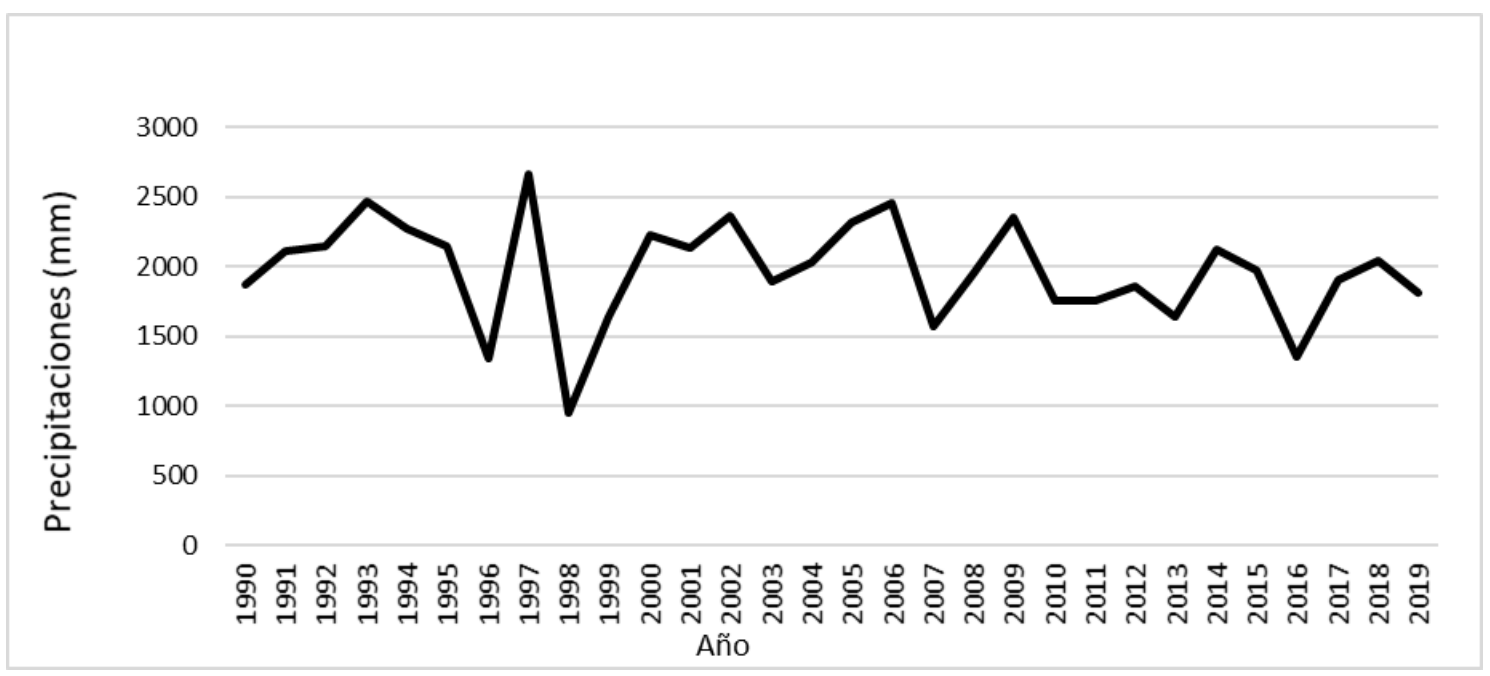

Figura 2. Precipitaciones anuales medidas en la estación pluviométrica Tricauco (China Muerta).

Fte.: Center for Climate and Resilience Research (2021). 


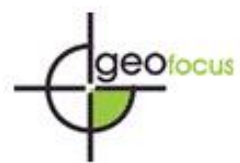

Peña M.A y Calderón P. (2021): "Evaluación de dos perturbaciones simultáneas en bosques de Arauca ria-Lenga, usando diferencias de indices espectrales", GeoFocus (Artículos), no 27, p. 5-18. http://dx.doi.org/10.21138/GF.689

El área de estudio se encuentra dentro de la zona climática afectada por la sequía referida en el acápite previo, lo cual queda expresado en la tendencia decreciente experimentada durante los últimos 10 años aproximadamente por las precipitaciones anuales medidas en la estación pluviométrica circundante a la Reserva (Figura 2).

\subsection{Selección y preparación de imágenes satelitales}

Cinco imágenes satelitales Landsat-8 OLI (operational land imager) del área de estudio fueron descargadas desde el buscador de imágenes en línea del Servicio Geológico de Estados Unidos (https://earthexplorer.usgs.gov/). La primera imagen, adquirida en el verano de 2013, representa la situación de la Reserva previa a las perturbaciones abordadas en este estudio, en tanto que las imágenes restantes corresponden a tres fechas de casi-aniversario adquiridas con frecuencia bianual dentro del período posterior a estas (Tabla 1).

Tabla 1. Fecha de adquisición de las imágenes satelitales empleadas.

\begin{tabular}{cc}
\hline Situación de la Reserva & $\begin{array}{c}\text { Fecha de adquisición de la } \\
\text { imagen }\end{array}$ \\
\hline Ex-ante & 24 de diciembre de 2013 \\
Ex-post & 30 de diciembre de 2015 \\
& 19 de diciembre de 2017 \\
& 25 de diciembre de 2019 \\
\hline
\end{tabular}

Las imágenes cuentan con una resolución espacial nominal de $30 \mathrm{~m}$ en todas sus bandas y corresponden al producto L1TP (Level 1 Terrain Precision); sujeto a calibración radiométrica y ortorectificación, por lo que para cumplir adecuadamente los objetivos del estudio, su radiometría en unidades de bits fue en primer lugar, convertida a radiancias absolutas, empleando la herramienta de calibración radiométrica del procesador de imágenes ENVI (ENvironment for Visualizing Images) 5.3○ (Exelis Visual Information Solutions Inc., Broomfield, EE.UU.). Posteriormente, estas radiancias fueron convertidas a reflectancias superficiales, usando el módulo FLAASH (Fast Line-of-sight Atmospheric Analysis of Hypercubes) del mismo software, el cual emplea el modelo de transferencia radiativa MODTRAN (Moderate Resolution Atmospheric Transmission, Spectral Sciences, Inc., Burlington, EE.UU.) para modelar la iluminancia que impacta la superficie terrestre (irradian cia superficial) y suprimir la radiación de ruta y adyacente añadida a la luminancia exoatmosférica (radiancia superficial) de la imagen.

\subsection{Delimitación de las áreas incendiada e infectada}

Para delimitar el área de estudio y sus tipos forestales, además de las áreas comprometidas por las perturbaciones de interés, fueron empleadas bases de datos vectoriales de la Reserva, provistas por el servicio en línea de Infraestructura de Datos Geoespaciales (http://www.ide.cl/) del Ministerio de Bienes Nacionales de Chile (IDE, 2021). Las especies forestales de la Reserva fueron agrupadas en función del tipo forestal, resultando dos grupos: (1) especies caducifolias de Nothofagus; Coigüe (nothofagus dombeyi (Mirb.) Oerst.), Coyán (nothofagus obliqua (Mirb.) Oerst.), Nirre (nothofagus antarctica (G. Forst.) Oerst)., Raulí (nothofagus alpina (Phil.) Dim. et Mil.), y en especial Lenga, con una superficie de 18,93 km² $(27 \%)$ y (2) comunidades de Araucarias como especie dominante, junto a otras especies secundarias también perennes, tales como Ciprés de la cordillera y Tepa, con una superficie de 


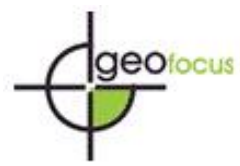

Peña M.A y Calderón P. (2021): "Evaluación de dos perturbaciones simultáneas en bosques de Arauca ria-Lenga, usando diferencias de indices espectrales", GeoFocus (Artículos), no 27, p. 5-18. http://dx.doi.org/10.21138/GF.689

$50,67 \mathrm{~km}^{2}(73 \%)$. El incendio comprometió $33,35 \mathrm{~km}^{2}$ de la Reserva $(39 \%)$, de los cuales $4,18 \mathrm{~km}^{2}(12.5 \%)$ corresponden al primer grupo, en adelante Lenga, y $25,07 \mathrm{~km}^{2}(75 \%)$ al segundo grupo, en adelante Araucaria. El área restante de $51,97 \mathrm{~km}^{2}(61 \%)$, se divide en $14,75 \mathrm{~km}^{2}(36,5 \%)$ correspondientes al grupo Lenga y $25,6 \mathrm{~km}^{2}(63,4 \%)$ correspondientes al grupo Araucaria (Tabla 2, Figura 3), definiendo éste último el área infectada, ya que la enfermedad comprometió directamente a esa especie. Como cabría suponer, la enfermedad también afectó a comunidades de Araucarias distribuidas al interior del área incendiada, sin embargo, estas fueron excluidas del área infectada debido a su muy posible confusión con el incendio como causa de perturbación.

Tabla 2. Superficie ocupada por las áreas perturbadas en la Reserva China muerta.

\begin{tabular}{|c|c|c|c|c|c|c|}
\hline \multirow{4}{*}{ Área } & \multicolumn{6}{|c|}{ Superficie } \\
\hline & \multirow{2}{*}{\multicolumn{2}{|c|}{$\begin{array}{c}\text { Total de la } \\
\text { Reserva }\end{array}$}} & \multicolumn{4}{|c|}{ Según grupo forestal } \\
\hline & & & \multicolumn{2}{|c|}{ Lenga } & \multicolumn{2}{|c|}{ Araucaria } \\
\hline & $\mathrm{km}^{2}$ & $\%$ & $\mathrm{~km}^{2}$ & $\%$ & $\mathrm{~km}^{2}$ & $\%$ \\
\hline Incendiada & 33,35 & 39 & 4,18 & 22 & 25,07 & 49 \\
\hline Infectada & 51,97 & 61 & 14,75 & 78 & 25,60 & 51 \\
\hline Total & 85,32 & 100 & 18,93 & 100 & 50,67 & 100 \\
\hline
\end{tabular}

Fte.: IDE (2021).

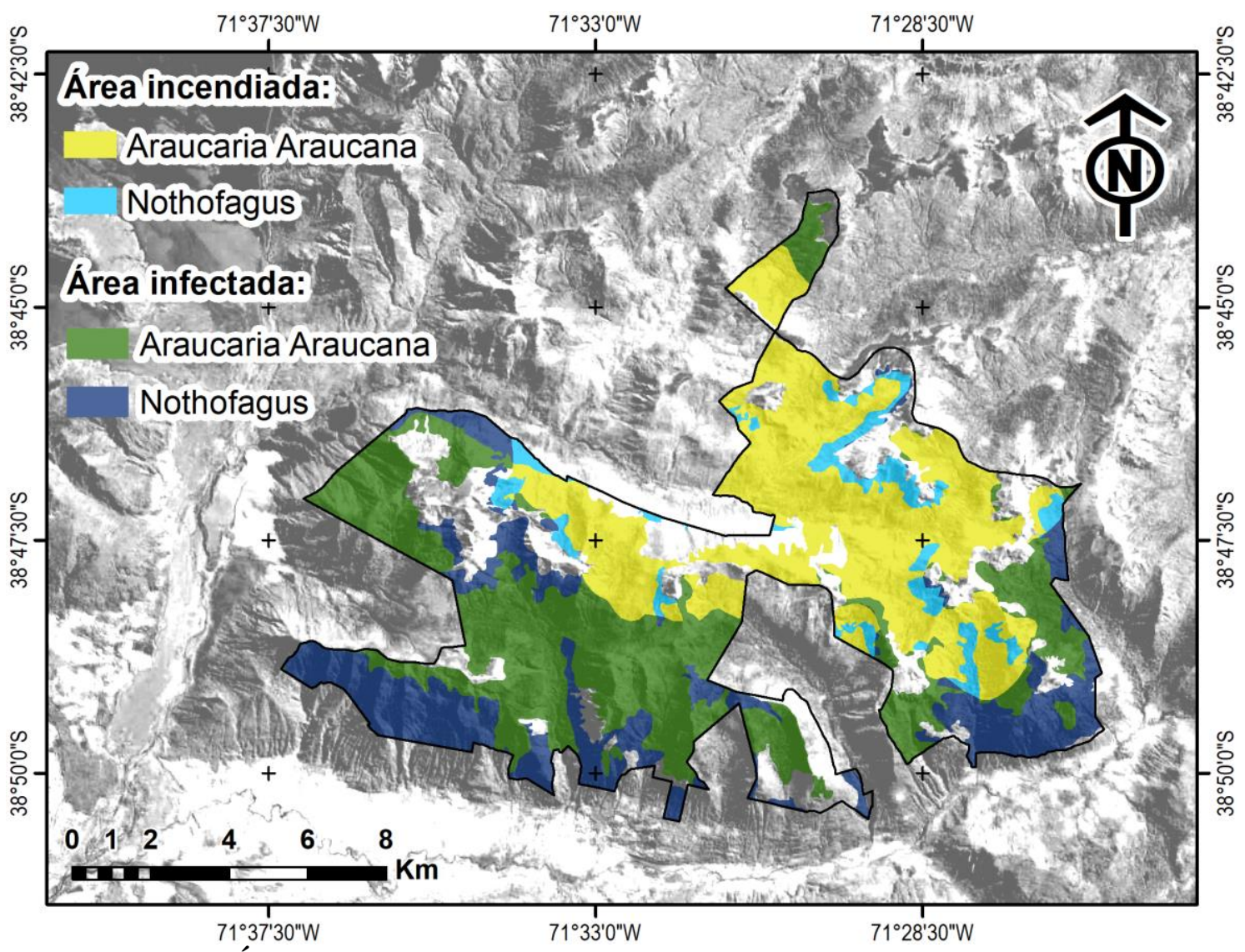

Figura 3. Áreas perturbadas en la Reserva China Muerta según tipo forestal.

Fte: IDE (2021). 
Peña M.A y Calderón P. (2021): "Evaluación de dos perturbaciones simultáneas en bosques de Arauca ria-Lenga, usando diferencias de indices espectrales", GeoFocus (Artículos), nº 27, p. 5-18. http://dx.doi.org/10.21138/GF.689

\subsection{Cálculo del índice de vigor y sus diferencias temporales}

En este estudio seleccionamos un único índice espectral de vegetación que fuera aplicable a ambas perturbaciones, para así favorecer la comparación de sus resultados. La literatura científica ofrece un gran número de estos, de acuerdo a la variable de estado vegetal que se desee estimar y a la fuente de distorsión radiométrica que se requiera controlar (Jones y Vaughan 2010, Xue y Su 2017). A este respecto, nos inclinamos por emplear una versión normalizada de la división entre las bandas del infrarrojo cercano (numerador) y rojo (denominador) de una imagen (ratio vegetation index), debido a su simpleza y robustez para retratar de manera integral el estado de vigor de la vegetación desde imágenes radiométricamente corregidas, independiente del disturbio que la afecte (Bannari et al. 1995).

$$
\mathrm{NVI}=\rho \mathrm{IC} /(\rho \mathrm{IC}+\rho \mathrm{R})
$$

(Ecuación 1)

Este índice, en adelante NVI (normalized vegetation index) es prácticamente idéntico al conocido NDVI, excepto que su formulación no incluye la resta de la banda del rojo $(\rho \mathrm{IC})$ a la del infrarrojo cercano en el numerador $(\rho \mathrm{IC})$ de la división, por lo que si bien los valores resultantes quedan constreñidos a una escala común, estos son solo positivos comprendidos entre 0 (suelos no vegetados) y $\sim 1$ (suelo saturado de vegetación sana). Esta escala facilitó la interpretación de los valores resultantes de la posterior sustracción efectuada entre el índice de la fecha ex-ante (minuendo) con cada una de las fechas ex-post (sustraendo) o $\Delta \mathrm{NVI}$, que menor valor indica una mayor similitud entre la condición ex-ante y ex-post de un píxel dado y por lo tanto, una mayor recuperación de vigor vegetal en el contexto del presente estudio.

\subsection{Confección de mapas}

A partir de los resultados arrojados por las diferencias temporales de índices anteriormente descritas, fueron elaborados dos mapas que sintetizan la trayectoria postperturbaciones de las coberturas afectadas: (1) recuperación de biomasa post-incendio y (2) deterioro de biomasa por infección. En ambos casos, para facilitar la interpretación espacial de del $\Delta \mathrm{NDI}_{2013-2019}$, sus valores fueron agrupados en tres rangos, cuyos umbrales fueron definidos para cada área afectada a partir de la suma y resta de desviaciones estándar sobre la media, un criterio estadístico de discretización de valores cuantitativos empleado en estudios similares (Escuín et al. 2008, Peña y Ulloa 2017).

\section{Resultados}

\section{1. Comportamiento del NVI en las áreas perturbadas}

Ambas áreas perturbadas muestran un elevado NVI promedio ex-ante (2013) (Tabla 3), que como cabría esperar, se reduce notoriamente una vez ocurrida o detectada cada perturbación (2015), aparejado a una mayor desviación estándar de sus valores. En los años posteriores (2017 y 2019), ambas áreas logran incrementar el promedio y reducir la desviación estándar de NVI, llegando el área infectada a casi equiparar sus estadísticos ex-ante (cabe destacar que los valores arrojados por el NVI no deben ser asimilables en términos absolutos a los del NDVI, por cuanto a causa de sus diferentes procedimientos de normalización, la escala del primero es más estrecha que la del segundo). 
Peña M.A y Calderón P. (2021): "Evaluación de dos perturbaciones simultáneas en bosques de Arauca ria-Lenga, usando diferencias de indices espectrales", GeoFocus (Artículos), no 27, p. 5-18. http://dx.doi.org/10.21138/GF.689

Tabla 3. Promedio y desviación estándar de NVI según año y tipo de perturbación.

\begin{tabular}{ccccc}
\hline \multicolumn{4}{c}{ NVI } \\
\hline \multirow{2}{*}{ Año } & \multicolumn{2}{c}{ Área } & \multicolumn{2}{c}{ Área } \\
incendiada & \multicolumn{2}{c}{ Infectada } \\
\cline { 2 - 5 } & $\overline{\mathrm{x}}$ & $\sigma$ & $\overline{\mathrm{x}}$ & $\sigma$ \\
\hline 2013 & 0,93 & 0,03 & 0,92 & 0,05 \\
2015 & 0,78 & 0,12 & 0,88 & 0,09 \\
2017 & 0,82 & 0,08 & 0,88 & 0,06 \\
2019 & 0,83 & 0,09 & 0,91 & 0,06 \\
\hline
\end{tabular}

Para el caso del área incendiada, es posible apreciar que las diferencias de los estadísticos de NVI según tipo forestal fueron muy menores (Tabla 4), razón por la que de aquí en adelante el área afectada por esta perturbación es tratada sin distinción del tipo forestal que la compone.

Tabla 4. Promedio y desviación estándar de NVI según año y tipo forestal del área incendiada.

\begin{tabular}{ccccc}
\hline \multicolumn{4}{c}{ NVI } \\
\hline \multirow{2}{*}{ Año } & \multicolumn{2}{c}{ Araucaria } & \multicolumn{2}{c}{ Lenga } \\
\cline { 2 - 5 } & $\bar{x}$ & $\sigma$ & $\bar{x}$ & $\sigma$ \\
\hline 2013 & 0,93 & 0,03 & 0,93 & 0,04 \\
2015 & 0,78 & 0,12 & 0,80 & 0,12 \\
2017 & 0,82 & 0,08 & 0,84 & 0,09 \\
2019 & 0,83 & 0,09 & 0,95 & 0,10 \\
\hline
\end{tabular}

\section{2. Comportamiento de las diferencias temporales de NVI en las áreas perturbadas}

En cuanto al área incendiada, se aprecia una disminución de 0,14 puntos en el promedio de $\Delta \mathrm{NVI}_{2013-2015}$ (Tabla 5), indicativa de la severidad del incendio en las coberturas vegetales de Lenga y Araucarias de la Reserva. La diferencia promedio del índice entre 2013 y 2017 destaca una recuperación de la biomasa calcinada 0,04 puntos mayor que la diferencia temporal anterior, mientras que el promedio de la tercera diferencia $\left(\Delta \mathrm{NVI}_{2013-2019}\right)$ solo logra incrementar esa recuperación en apenas 0,01 puntos, lo que denota la ralentización del ritmo de recuperación de la biomasa afectada por esta perturbación entre 2015 y 2019.

Tabla 5. Promedio y desviación estándar de las diferencias temporales de NVI según tipo de perturbación.

\begin{tabular}{|c|c|c|c|c|}
\hline \multicolumn{5}{|c|}{$\Delta \mathrm{NVI}$} \\
\hline \multirow{2}{*}{$\begin{array}{l}\text { Diferencia } \\
\text { temporal }\end{array}$} & \multicolumn{2}{|c|}{$\begin{array}{c}\text { Área } \\
\text { incendiada }\end{array}$} & \multicolumn{2}{|c|}{$\begin{array}{c}\text { Área } \\
\text { Infectada }\end{array}$} \\
\hline & $\bar{x}$ & $\sigma$ & $\bar{x}$ & $\sigma$ \\
\hline $2013-2015$ & 0,14 & 0,11 & 0,03 & 0,09 \\
\hline 2013-2017 & 0,10 & 0,07 & 0,04 & 0,04 \\
\hline 2013-2019 & 0,09 & 0,08 & 0,01 & 0,02 \\
\hline
\end{tabular}




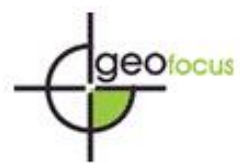

Peña M.A y Calderón P. (2021): "Evaluación de dos perturbaciones simultáneas en bosques de Arauca ria-Lenga, usando diferencias de indices espectrales", GeoFocus (Artículos), no 27, p. 5-18. http://dx.doi.org/10.21138/GF.689

En cuanto al área infectada, la disminución en el promedio de $\Delta \mathrm{NVI}_{2013-2015}$ fue de 0,03 puntos (Tabla 5), es decir, mucho menor que en el caso del incendio para la misma diferencia temporal del índice. Posteriormente, el promedio de $\Delta \mathrm{NVI}_{2013-2017}$ se elevó en 0,01 puntos con respeto a la diferencia anterior, para luego reducirse casi a cero en el período 2013-2019, todo lo cual refleja el menor impacto que produjo el brote infeccioso en comparación con el incendio, sobre la abundancia de biomasa de los bosques de la Reserva.

\subsection{Mapa de recuperación de biomasa post-incendio}

La Figura 4 muestra la recuperación relativa que ha experimentado la biomasa forestal incendiada al interior de la Reserva entre 2015 y 2019. La clase mayor abarca áreas cuyo $\Delta \mathrm{NVI}_{2013-2019}$ está bajo el valor promedio, es decir, exhiben una alta recuperación en relación con el estado de su biomasa previo al incendio. La clase de recuperación moderada comprende áreas cuyo $\Delta \mathrm{NVI}_{2013-2019}$ está entre el promedio y una desviación estándar de sus valores, mientras que la clase de recuperación menor abarca áreas cuyo $\Delta \mathrm{NVI}_{2013-2019}$ está por sobre el promedio más una desviación estándar, por lo que muestran la menor recuperación postincendio. La Figura ratifica lo expuesto por Urrutia et al. (2018), esto es: un patrón de distribución espacial dominante del incendio en dirección noreste; propiciado por vientos provenientes desde el Pacífico con dirección a la Cordillera de Los Andes, así como la presencia de una mayor recuperación de biomasa en zonas bajas, que se vieron menos afectadas por el fuego que las zonas altas; caracterizadas por una mayor densidad arbórea.

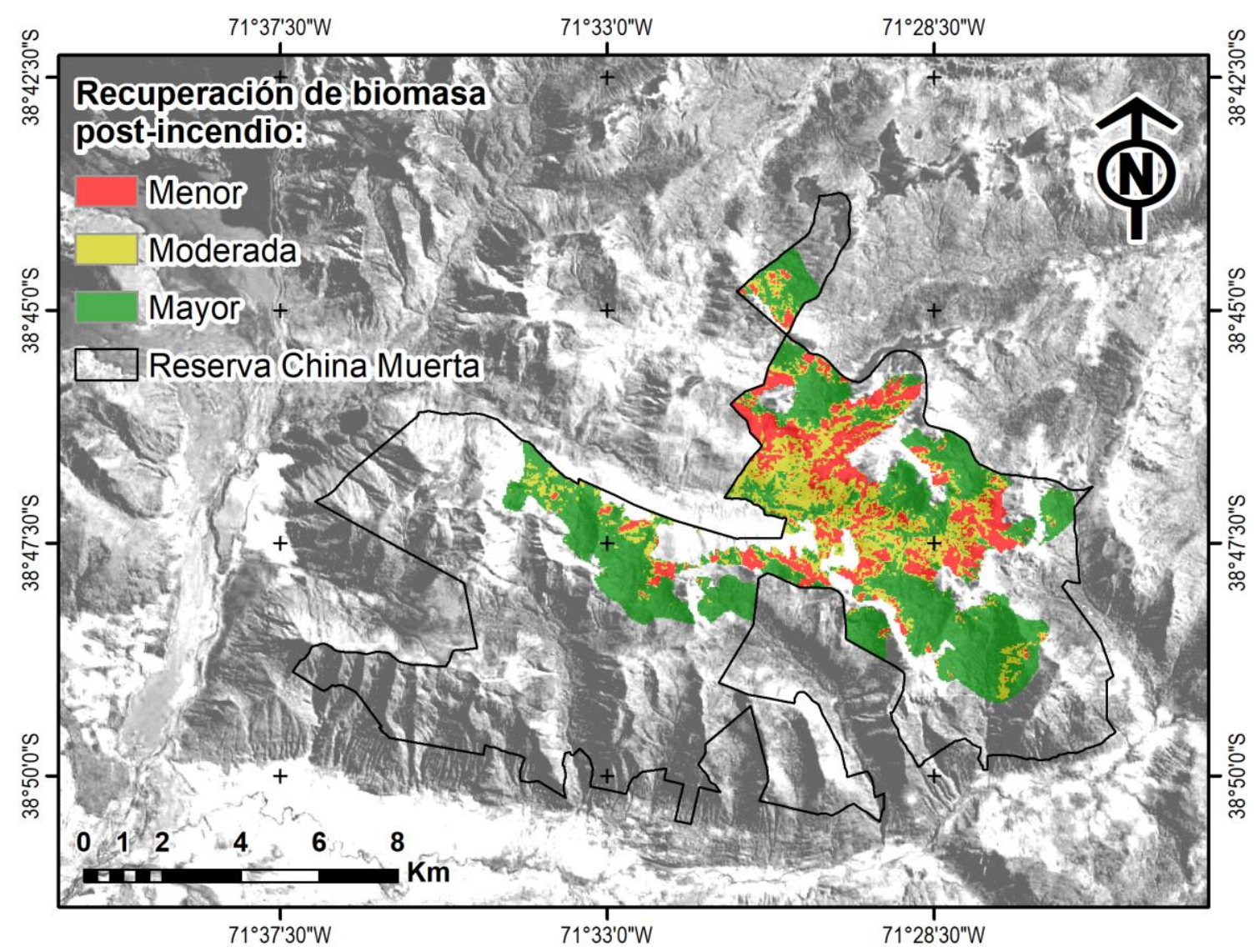

Figura 4. Clases de recuperación de biomasa post-incendio entre 2015 y 2019. 


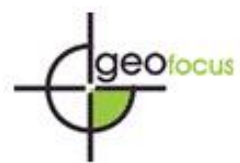

Peña M.A y Calderón P. (2021): "Evaluación de dos perturbaciones simultáneas en bosques de Arauca ria-Lenga, usando diferencias de indices espectrales", GeoFocus (Artículos), no 27, p. 5-18. http://dx.doi.org/10.21138/GF.689

\subsection{Mapa de deterioro de biomasa por infección}

La Figura 5 muestra el nivel de deterioro relativo que ha experimentado la biomasa forestal al interior de la Reserva entre 2015 y 2019 producto de la infección. La clase de deterioro menor comprende áreas cuyo $\Delta \mathrm{NVI}_{2013-2019}$ está bajo el valor promedio siendo incluso en algunos casos negativo, es decir, exhiben una alta recuperación o incluso han mejorado en relación con el estado de su biomasa previo a la detección del brote de la enfermedad. La clase de deterioro moderado comprende áreas cuyo $\Delta \mathrm{NVI}_{2013-2019}$ está entre el promedio y una desviación estándar, mientras que la clase de deterioro alto abarca áreas cuyo $\Delta \mathrm{NVI}_{2013-2019}$ está por sobre el promedio más una desviación estándar, por lo que muestran el mayor deterioro de biomasa por infección. La misma Figura permite apreciar con claridad la distribución espacial sur de la infección, destacando la predilección del patógeno a asentarse en zonas húmedas (Vélez et al. 2018)

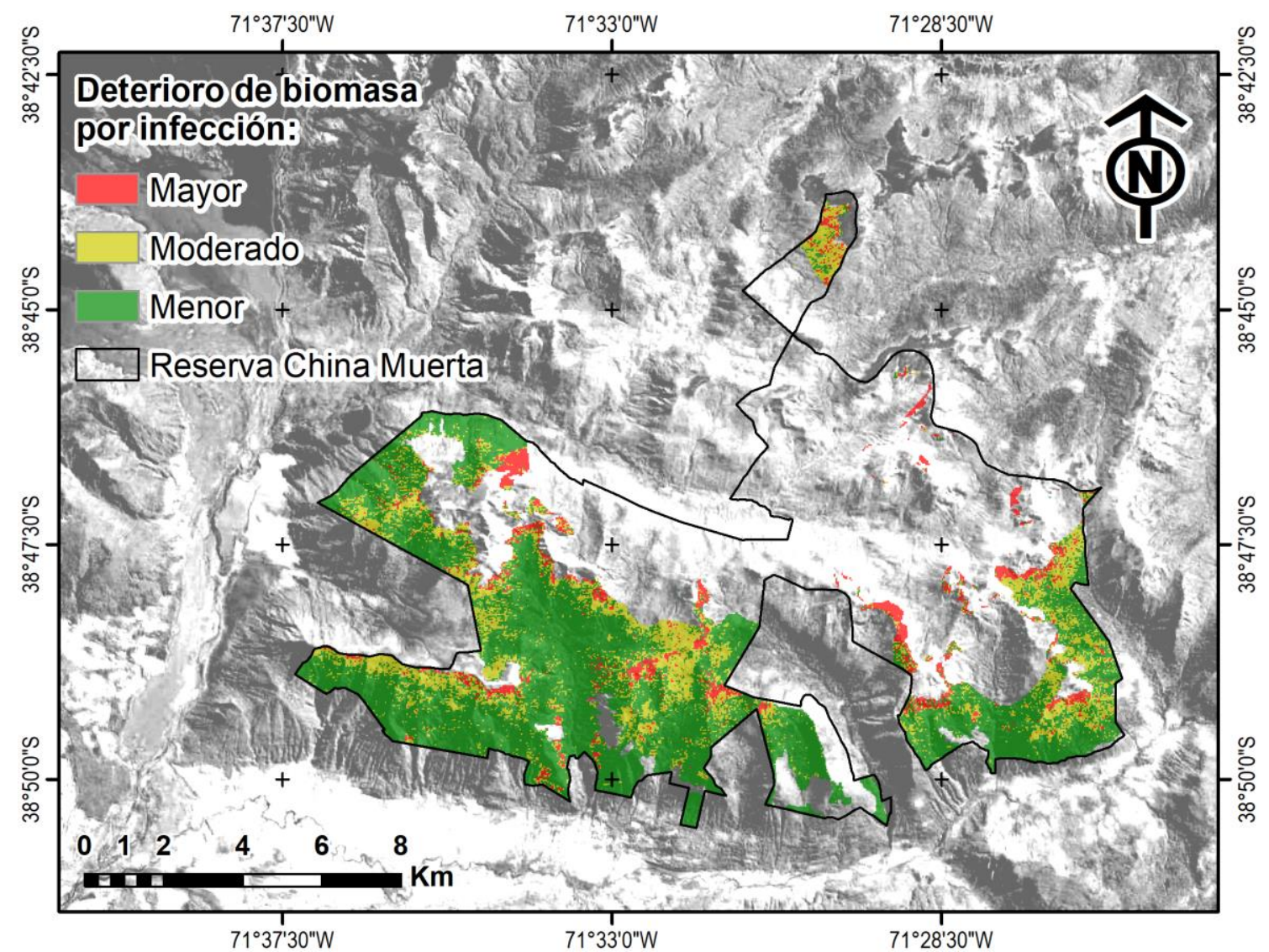

Figura 5. Clases de deterioro de biomasa por infección entre 2015 y 2019.

En cuanto a la superficie que cubren las clases definidas para cada mapa (Tabla 6), es posible apreciar que aquellas que representan la mayor recuperación o menor afectación, según sea la perturbación considerada, son las más predominantes dentro de las respectivas áreas afectadas, alcanzando un $57 \%$ en el caso del incendio y un $67 \%$ en el caso de la infección. 
Peña M.A y Calderón P. (2021): "Evaluación de dos perturbaciones simultáneas en bosques de Arauca ria-Lenga, usando diferencias de indices espectrales", GeoFocus (Artículos), $n^{\circ}$ 27, p. 5-18. http://dx.doi.org/10.21138/GF.689

Tabla 6. Superficie ocupada por las clases de recuperación post-incendio y de deterioro de biomasa por infección entre 2015 y 2019.

\begin{tabular}{crrcrr}
\hline \multicolumn{2}{c}{ Área incendiada } & \multicolumn{3}{c}{ Área infectada } \\
\hline $\begin{array}{c}\text { Clase de } \\
\text { recuperación }\end{array}$ & $\mathrm{km}^{2}$ & $\%$ & $\begin{array}{c}\text { Clase de } \\
\text { afectación }\end{array}$ & $\mathrm{km}^{2}$ & $\%$ \\
\hline Menor & 5,5 & 19 & Mayor & 3,44 & 9 \\
Moderado & 7,13 & 24 & Moderado & 9,90 & 24 \\
Mayor & 16,86 & 57 & Menor & 27,31 & 67 \\
Total & 29,49 & 100 & Total & 40,65 & 100 \\
\hline
\end{tabular}

\section{Discusión y conclusiones}

Este estudio revela el impacto relativo producido por dos perturbaciones recientes que han afectado simultáneamente el estado de bosques de Araucaria-Lenga, ubicados en la Reserva China Muerta, Chile, mediante la detección de la recuperación o deterioro de su biomasa a partir de la resta entre fechas ex-ante y ex-post de un índice espectral de vigor vegetal. A este respecto, se observa que si bien tanto las áreas incendiadas como infectadas exhibieron un similar promedio de NVI ex-ante, una vez desencadenadas las perturbaciones el incendio comportó un mayor daño sobre la biomasa forestal; expresado en su mayor promedio de $\triangle \mathrm{NVI}_{2013-2015}$, además de una menor resiliencia a lo largo del período de interés; expresada en sus mayores promedios de $\Delta \mathrm{NVI}_{2013-2017}$ y $\Delta \mathrm{NVI}_{2013-2019}$. Este resultado connota la manifestación más repentina y agresiva de este fenómeno en comparación con la infección, que si bien se caracteriza por transcurrir de forma más gradual en el tiempo, suele ser más pervasiva, haciendo que los ecosistemas afectados tarden tiempos relativamente amplios en alcanzar su estado exante (Jones y Vaughan 2010). Si bien la interpretación biofísica de la magnitud de la severidad del incendio aquí obtenida (mediante el promedio de $\Delta \mathrm{NVI}_{2013-2015}$ ) se ve limitada a causa de provenir de una resta de índices espectrales inherentemente adimensionales que no pretendió ser sujeta a un modelado empírico, su comparación con aquella obtenida por Peña y Martínez (2019) en un incendio forestal de singular voracidad (conocido como Nilahue-Barahona) que afectó recientemente a Chile central, facilita su dimensionamiento. Los autores emplearon un a escala normalizada entre 0 y 1 del NDVI, que resulta ser equivalente a la del NVI aquí empleado, obteniendo una diferencia promedio de 0,24 entre sus fechas ex-ante y ex-post, es decir, una magnitud de severidad del incendio superior en 0,1 a la arrojada por el promedio de $\Delta \mathrm{NVI}_{2013-2015}$ del incendio de la Reserva China Muerta. Tal diferencia puede explicarse por la característica alta carga de combustible que caracteriza a las plantaciones forestales que mayoritariamente conforman el área de Nilahue-Barahona, así como por la presencia de las inusitadas condiciones de calor y sequedad que presentó el clima Mediterráneo allí imperante, situación que contrasta con la menor carga de combustible asociada a las especies nativas de la Reserva China Muerta, así como con el clima templado en que se inserta. Por otro lado, cabe señalar que mientras la imagen ex-post de Nilahue-Barahona fue adquirida antes de un mes de finalizado el incendio, la empleada en China Muerta fue adquirida ocho meses después de finalizado este, es decir, cuando al menos la vegetación de menor envergadura, experimentó algún grado de recuperación.

El estudio realizado por Vélez et al. (2018) sobre poblaciones de Araucarias chilenoargentinas, reveló que durante las últimas décadas éstas han experimentado un proceso de decaimiento inducido por el cambio climático (expresado en el aumento de temperaturas, la disminución de las precipitaciones y la consecuente expansión del rango geográfico de especies invasoras), produciendo en la especie una "hambruna del carbono" que le ha significado una mayor predisposición al ataque de patógenos. Dentro de éstos, destaca el oomicete, cuyo daño a 
Peña M.A y Calderón P. (2021): "Evaluación de dos perturbaciones simultáneas en bosques de Arauca ria-Lenga, usando diferencias de indices espectrales", GeoFocus (Artículos), no 27, p. 5-18. http://dx.doi.org/10.21138/GF.689

nivel de dosel en la especie se expresa en la progresión acrópeta de síntomas de clorosis, enrojecimiento, necrosis y defoliación parcial y aleatoria en su follaje, situación que si bien deriva esporádicamente en la muerte del individuo, puede ralentizar de manera importante su regeneración, afectando a la postre la dinámica poblacional de las Araucarias. Cabe esperar entonces, que estos síntomas se expresen desde la planimetría propia de una imagen como una merma en la vigorosidad de la cobertura vegetal retratada, la que efectivamente es constatada en este estudio mediante el uso de diferencias temporales de NVI.

La Araucaria ha evolucionado en ambientes volcánicamente activos, proclives a generarle estrés hídrico y nutricional por acción del calor/fuego y empobrecimiento del suelo, llevándola a generar respuestas adaptativas como una gruesa corteza, protección de yemas apicales, lenta renovación de hojas, raíces extensas y con alta capacidad de rebrote, además de mecanismos como la producción de ABA. No obstante, los bosques de Araucaria-Lenga son vulnerables a incendios severos, que suelen ocasionarse luego de veranos secos y cálidos precedidos de eventos El Niño, que afectan cíclicamente el área de estudio (González y Veblen 2006). Si bien las especies de Nothofagus que componen a este tipo forestal tradicionalmente han mostrado una buena capacidad de recolonización luego de incendios severos (González y Veblen 2007), el estudio de Urrutia et al. (2018) llevado a cabo sobre las comunidades forestales incendiadas en la Reserva China Muerta, destacó que la recuperación de poblaciones de Lenga específicamente, podría verse más dificultada, ya que para su reproducción la especie necesita de una fuente de semillas cercana, así como de un estrato arbóreo que promueva y proteja su establecimiento. Sumado a esto, los autores constataron un rápido cambio composicional en los bosques de Araucaria-Lenga, evidenciado en una reducción de su riqueza florística ex-post, así como en el reemplazo de especies herbáceas y del sotobosque por colonizadoras más tolerantes al fuego.

Estudios previos han consignado que bosques moderadamente a severamente dañados por un incendio, han mostrado una disminución notoria en sus diferencias temporales de índices de vegetación hacia el tercer año ex-post, aproximándose a sus niveles primigenios de biomasa a partir del séptimo año (Díaz-Delgado et al. 2003, Bisson et al. 2008, Chen et al. 2011). Si bien existen múltiples factores caso-específicos que advierten poner cautela en la transferibilidad de tales hallazgos, como por ejemplo la naturaleza nativa o introducida del bosque en estudio, estos sugieren que en nuestra área de estudio cabría esperar una importante contracción de las diferencias temporales de NVI a partir de los próximos dos o tres años (2020-2022). A pesar de esto, y sobre la base de los hallazgos de Urrutia et al. (2018) anteriormente expuestos, cabe conjeturar que aunque estas coberturas lograran luego de un tiempo restablecer sus niveles de biomasa previos a la perturbación, exhibirán una composición y estructura vegetal distinta a la primigenia, la cual no es posible de rastrear únicamente mediante índices espectrales de vegetación. Por tal motivo, estudios futuros referidos al seguimiento de la recuperación postperturbación de estos bosques deberían apoyarse en datos de campo que permitan medir la recuperación de esos y otros atributos ecológicos.

Este estudio empleó una metodología de evaluación post-perturbación de relativa fácil implementación en cualquier ecosistema vegetal, requiriendo básicamente de la correcta selección y corrección radiométrica de imágenes ópticas satelitales y su combinación con capas temáticas vectoriales de las coberturas vegetales de interés. Esto la hace fácilmente replicable y actualizable, confiriéndole una ventaja por sobre otros métodos de trazabilidad de recuperación de ecosistemas perturbados, razón que soporta la difusión de un estudio como este en pos de servir de referencia para su implementación en numerosos ecosistemas vegetales chilenos perturbados que aún no cuentan con apropiadas metodologías de monitoreo. 
Peña M.A y Calderón P. (2021): "Evaluación de dos perturbaciones simultáneas en bosques de Arauca ria-Lenga, usando diferencias de índices espectrales", GeoFocus (Artículos), no 27, p. 5-18. http://dx.doi.org/10.21138/GF.689

\section{Referencias bibliográficas}

Bannari, A., Morin, D., Bonn, F. y Huete, A.R. (1995): “A review of vegetation indices", Remote Sensing Reviews, 13(1-2), 95-120.

Banskota, A., Kayastha, N., Falkowski, M. J., Wulder, M. A., Froese, R. E. y White, J. C. (2014): "Forest Monitoring Using Landsat Time Series Data: A Review", Canadian Journal of Remote Sensing, 40, 362-364.

Bisson, M., Fornaciai, A., Coli, A., Mazzarini, F. y Pareschi, M. T. (2008): “The vegetation resilience after fire (VRAF) index: development, implementation and an illustration from central Italy", International Journal of Applied Earth Observation and Geoinformation, 10, 312-329.

Center for Climate and Resilience Research (2021): Mawún: explorador de precipitaciones. http://www.cr2.cl/mawun-explorador-de-precipitaciones/ (consultado 10-01-21).

Chuvieco, E. (1995): Fundamentos de teledetección espacial, Madrid, Ediciones RIALP, S.A.

Chuvieco, E., Martín, M. P. y Palacios, A. (2002): “Assessment of different spectral indices in the red-near-infrared spectral domain for burned land discrimination", International Journal of Remote Sensing, 23, 5103-5110.

Chen, X., Vogelmann, J. E., Rollins, M., Ohlen, D., Key, C. H., Yang, L., Huang, C., Shi, H. (2011): "Detecting post-fire burn severity and vegetation recovery using multitemporal remote sensing spectral indices and field-collected composite burn index data in a ponderosa pine forest", International Journal of Remote Sensing, 32(23), 7905-7927.

CONAF (Corporación Nacional Forestal) (2014): Plan de Manejo Reserva Forestal China Muerta, Santiago, Oficina Central de CONAF.

De la Barrera, F. y Ruiz, V. (2017): "Evaluación del impacto de los incendios de Chile CentroSur en el verano del año 2017", IALE-Chile, 1, 1-2.

Díaz-Delgado, R., Lloret, F. y Pons, X. (2003): "Influence of fire severity on plant regeneration by means of remote sensing imagery", International Journal of Remote Sensing, 24(8), 17511763.

Escuín, S., Navarro, R. y Fernández, P. (2008): "Fire severity assessment by using NBR (normalized burn ratio) and NDVI (normalized difference vegetation index) derived from Landsat TM/ETM images", International Journal of Remote Sensing, 29(4), 1053-1073.

Figueroa-Burdiles, N. y Vergara-Pinto, F. (2018): "Reserva Nacional China Muerta: Consideraciones en torno a la conservación biocultural de la naturaleza, los incendios forestales y la herida colonial en territorios indígenas", Cultura-Hombre-Sociedad, 28, 103-110.

Frolking, S., Palace, M. W., Clark, D. B., Chambers, J. Q., Shugart, H. H. y Hurtt, G. C. (2009): "Forest disturbance and recovery: A general review in the context of spaceborne remote sensing of impacts on aboveground biomass and canopy structure", Journal of Geophysical Research, $114,2-20$.

Fuentes-Ramírez, A., Vargas-Arroyo, P., Del Fierro, A. y Pérez, F. (2019): "Post-fire response of Araucaria araucana (Molina) K. Koch: Assessment of vegetative resprouting, seed production and germination", Gayana Botánica, 76, 119-121.

Gajardo, R. (1994): La vegetación natural de Chile: clasificación y distribución geográfica. Santiago, Editorial Universitaria. 


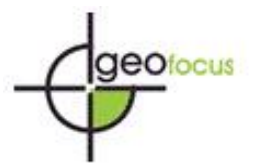

Peña M.A y Calderón P. (2021): "Evaluación de dos perturbaciones simultáneas en bosques de Arauca ria-Lenga, usando diferencias de indices espectrales", GeoFocus (Artículos), no 27, p. 5-18. http://dx.doi.org/10.21138/GF.689

González, M. E. y Veblen, T. T. (2006): "Climatic influences on fire in Araucaria-Nothofagus forests in the Andean cordillera of south-central Chile", Ecoscience, 13, 343-345.

González, M. E. y Veblen, T. T. (2007): "Incendios en bosques de Araucaria araucana y consideraciones ecológicas al madereo de aprovechamiento en áreas recientemente quemadas", Revista Chilena de Historia Natural, 80, 244-248.

González, M. E., Gómez-González, S., Lara, A., Garreaud, R. y Díaz-Hormazábal, I. (2018): "The 2010-2015 Megadrought and its influence on the fire regime in central and south-central Chile", Ecosphere, 9, 2-3.

Hirschmugl, M., Gallaun, H., Dees, M., Datta, P., Deutscher, J., Koutsias, N. y Schardt, M. (2017): "Methods for mapping forest disturbance and degradation from optical Earth observation data: a review", Current Forestry Reports, 3, 1-11.

IDE (Infraestructura de Datos Espaciales) (2021). Visor de Mapas de la IDE Chile.

http://www.ide.cl/ (consultado 10-01-21).

Jones, H. y Vaughan, R. (2010): Remote sensing of vegetation: principles, techniques and applications, New York, Oxford University Press.

Kushla, J. D., y Ripple, W. J. (1998): “Assessing wildfire effects with Landsat thematic mapper data", International Journal of Remote Sensing, 19, 2493-2504.

Lusk, C. H. (2001): "Leaf life spans of some conifers of the temperate forests of South America", Revista Chilena de Historia Natural, 74, 711-712.

Peña, M. A. y Martínez, G. (2019): "Evaluación del impacto del incendio Nilahue-Barahona mediante índices espectrales ex-ante y ex-post", Revista Geográfica de Chile Terra Australis, $55,1-11$.

Peña, M. A. y Ulloa, J. (2017): "Mapeo de la recuperación de la vegetación quemada mediante la clasificación de índices espectrales pre- y post-incendio", Revista Asociación Española de Teledetección, 50, 37-45.

Raffaele, E., De Torres Curth, M., Morales, C. L. y Kitzberger, T. (2014): Ecología e historia natural de la Patagonia Andina: un cuarto de siglo de investigación en biogeografía. Buenos Aires, Fundación de Historia Natural Félix de Azara.

Townsend P. A., Singh, A., Foster, J. R., Rehberg, N. J., Kingdon, C. C., Eshlem an, K. N., Seagle, S. W. (2012): "A general Landsat model to predict canopy defoliation in broadleaf deciduous forests", Remote Sensing of Environment, 119, 255-265.

Urrutia-Estrada, J., Fuentes-Ramírez, A. y Hauenstein, E. (2018): "Diferencias en la composición florística en bosques de Araucaria-Nothofagus afectados por distintas severidades de fuego", Gayana Botánica, 75, 625-634.

Vélez, M. L., Salgado, M. E., Marfetan, J. A., Tirante, S. I., Mattes H., Ávila, M., Szchowski, A., Viviani, D. y Sanguinetti, J. (2017): Caracterización de la desecación del dosel y sanidad de Araucaria Araucana en Argentina. Buenos Aires, Sanguinetti, J. Editores.

Xue, J. y Su, B. (2017): "Significant remote sensing vegetation indices: A Review of Developments and Applications", Journal of Sensors, 2017: 1-17 